\title{
The complexity of titin splicing pattern in human adult skeletal muscles
}

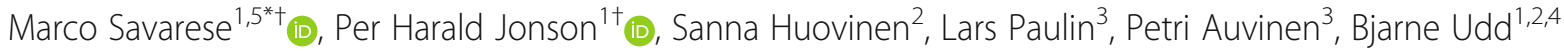 \\ and Peter Hackman ${ }^{1}$
}

\begin{abstract}
Background: Mutations in the titin gene (TTM) cause a large spectrum of diseases affecting skeletal and/or cardiac muscle. TTN includes 363 coding exons, a repeated region with a high degree of complexity, isoform-specific elements, and metatranscript-only exons thought to be expressed only during fetal development. Although three main classes of isoforms have been described so far, alternative splicing events (ASEs) in different tissues or in different developmental and physiological states have been reported.

Methods: To achieve a comprehensive view of titin ASEs in adult human skeletal muscles, we performed a RNASequencing experiment on 42 human biopsies collected from 12 anatomically different skeletal muscles of 11 individuals without any skeletal-muscle disorders.

Results: We confirmed that the skeletal muscle N2A isoforms are highly prevalent, but we found an elevated number of alternative splicing events, some at a very high level. These include previously unknown exon skipping events and alternative $5^{\prime}$ and $3^{\prime}$ splice sites. Our data suggests the partial inclusion in the TTN transcript of some metatranscript-only exons and the partial exclusion of canonical N2A exons.
\end{abstract}

Conclusions: This study provides an extensive picture of the complex TTN splicing pattern in human adult skeletal muscle, which is crucial for a proper clinical interpretation of TTN variants.

Keywords: Titin, Titinopathies, RNA-sequencing, Exon usage, Alternative splicing events, Splicing pattern

\section{Background}

The TTN gene encodes titin, a muscle protein spanning from the Z-disk to the M-band within the sarcomere. The genomic structure of TTN is quite remarkable. It contains 364 exons (363 coding exons plus the first non-coding exon) and can theoretically generate more than one million splice variants $[1,2]$. It also has a large repeated region with a high degree of complexity [1].

Titin isoforms have traditionally been classified in three main categories based on the presence of the N2A and N2B elements within the I-band region [3-5]. N2A isoforms (mainly expressed in the skeletal muscles) contain the N2A element, but not the cardiac-specific N2B element. On the contrary, N2B isoforms only

\footnotetext{
* Correspondence: marco.savarese@helsinki.fi

${ }^{\dagger}$ Equal contributors

${ }^{1}$ Folkhälsan Research Center, University of Helsinki, Helsinki, Finland

${ }^{5}$ Folkhälsan Institute of Genetics, Department of Medical Genetics, University

of Helsinki, Biomedicum, Haartmaninkatu 8, Pb 63, 00014 Helsinki, Finland

Full list of author information is available at the end of the article
}

include the cardiac-specific N2B element. N2BA isoforms, expressed in the heart, include both the N2B and N2A elements. N2A and N2BA isoforms also include additional exons, resulting in a higher number of Ig and PEVK domains in the I-band region.

Two further isoforms, named Novex-1 and Novex-2, are very similar to N2B but each of them includes an isoformspecific exon (exon 45 and exon 46, respectively). Finally, the Novex-3 isoform only contains the N-terminal part of titin due to an alternative stop codon in the Novex-3specific exon 48.

Interestingly, specific exons included in the inferred complete metatranscript (NM_001267550.1) and referred to as metatranscript-only or meta-only exons are thought to be expressed only during embryonic development. Thereafter, they are not included in the canonical soleusderived N2A skeletal muscle isoform, or in any of the five cardiac isoforms. 
An extensive use of alternative splicing (AS) in different tissues or in different developmental and physiological states has been reported, resulting in a longer or smaller protein [2]. This reflects the global massive use of tissue-specific AS events (ASEs) which have been described in the skeletal muscle $[6,7]$.

Although the presence of multiple different transcripts originating from TTN gene as consequence of ASEs has been partly suggested by experimental evidence $[1,2,8]$, we still lack a clear picture of the global exon usage and of the subsequent splicing profile of TTN muscular transcripts.

The introduction of RNA sequencing (RNA-Seq) methods has enabled a comprehensive study of the transcriptome [9]. Although early work focused on gene-expression analyses, RNA-Seq is a powerful tool for the identification and the study of alternative exon and splice site usage and of novel isoforms. It also allows an accurate quantification of relative transcript abundances $[6,10]$.

In this study, we analyzed RNA sequencing data of human adult skeletal muscle tissues to obtain a comprehensive view of titin ASEs. This is crucial for a proper clinical interpretation of TTN variants that have been associated with a wide spectrum of human diseases and for an improved genotype-phenotype correlation [11-16].

\section{Methods}

\section{Skeletal muscle samples and RNA extraction}

Data was generated using 42 human skeletal muscle samples dissected from 12 anatomically different skeletal muscles (tibialis anterior, flexor hallucis longus, soleus, extensor digitorum longus, gracilis, semitendinosus, semimembranosus, vastus medialis, vastus lateralis, sartorius, biceps femoris, adductor magnus) collected from 11 adult individuals (7 males and 4 females) who had undergone above or below-the-knee amputation surgery for medical reasons other than neuromuscular disorders (Additional file 1:Table S1). A written informed consent was signed by all the patients and the Tampere University Hospital (Tampere, Finland) Ethics Committee approved the study.

The samples $(5 \times 5 \mathrm{~mm}$ of size $)$ were processed immediately after their removal to avoid tissue degradation as previously described [17]. Total RNA was extracted from the selected samples by the TRIzol reagent method, according to the manufacturer's instructions (Invitrogen, Life Technologies, Canada). RNA quality was checked with BioAnalyzer equipment using the RNA 6000 Nano Assay kit (Agilent Technologies, CA, USA).

\section{Library preparation, sequencing, and bioinformatics}

Indexed sequencing libraries were generated from $1 \mu \mathrm{g}$ of total RNA, using the TruSeq Stranded Total RNA kit according to the manufacturer's instructions (Illumina,
CA, USA). Single-end sequencing (86 bp reads) of multiplex libraries was performed on NextSeq500 instrument. Raw reads were mapped against the hg19 human reference genome using TopHat2 [18]. TopHat was also used for detecting and counting exon junctions. Alternative splice sites were evaluated using $\mathrm{Hu}$ man Splicing Finder (HSF) program [19].

For each exon, the inclusion rate was calculated as $[(I / 2) /[(I / 2)+E]$, where $I$ is the number of reads supporting the exon inclusion (all junctions going into and exiting the exon) and $E$ is the number of reads supporting its exclusion.

\section{Experimental validation of alternative splicing events}

For experimental validation of RNA-Seq results, cDNA synthesis was performed using SuperScript III First-Strand Synthesis System (Thermo Scientific, USA). RT-PCRs were performed using $1 \mu \mathrm{l}$ of cDNA and a DreamTaq ${ }^{\text {ma }}$ DNA Polymerase (Thermo Scientific). Primers were designed with Primer3 software (sequences available upon request). Amplified products were separated on $2 \%$ agarose gels and specific electrophoresis bands, corresponding to differently spliced products, were extracted using NucleoSpin Gel and PCR Clean-up (Macherey-Nagel, Germany) and analyzed by Sanger sequencing.

\section{Publicly available data}

We also evaluated the presence of the ASEs in publicly available total mRNA sequencing data of adult gastrocnemius medialis from the ENCODE project (https://www.encodeproject.org; accession numbers ENCFF219LYV, ENCFF308RYZ, ENCFF569TCU, ENCFF408QZN, and ENCFF064NBB). Junctions were extracted from the available bam-files using regtools (https://github.com/griffithlab/regtools).

Similarly, we analyzed RNA-Seq data from fetal skeletal muscles (accession numbers ENCFF009MKH, ENCFF084FDS, ENCFF121PKV, and ENCFF405BHX), fetal heart (accession numbers ENCFF111DKK, ENCFF686KAP, ENCFF167WVS, and ENCFF174EGJ), and adult heart (accession numbers ENCFF735RZM, ENCFF834OIQ, ENCFF608FZD, and ENCFF621SXE) from ENCODE.

\section{Results}

Before focusing on alternative splice events, we analyzed the canonical junctions, which are present in the previously reported isoforms, to evaluate their relative expression in human adult skeletal muscles. As expected, the junction 47-50, uniquely present in the previously identified skeletal long isoform N2A [1], is detected at very high level in all our samples (Fig. 1a). This confirms that most of the skeletal muscle transcripts belong to this class of isoforms. 


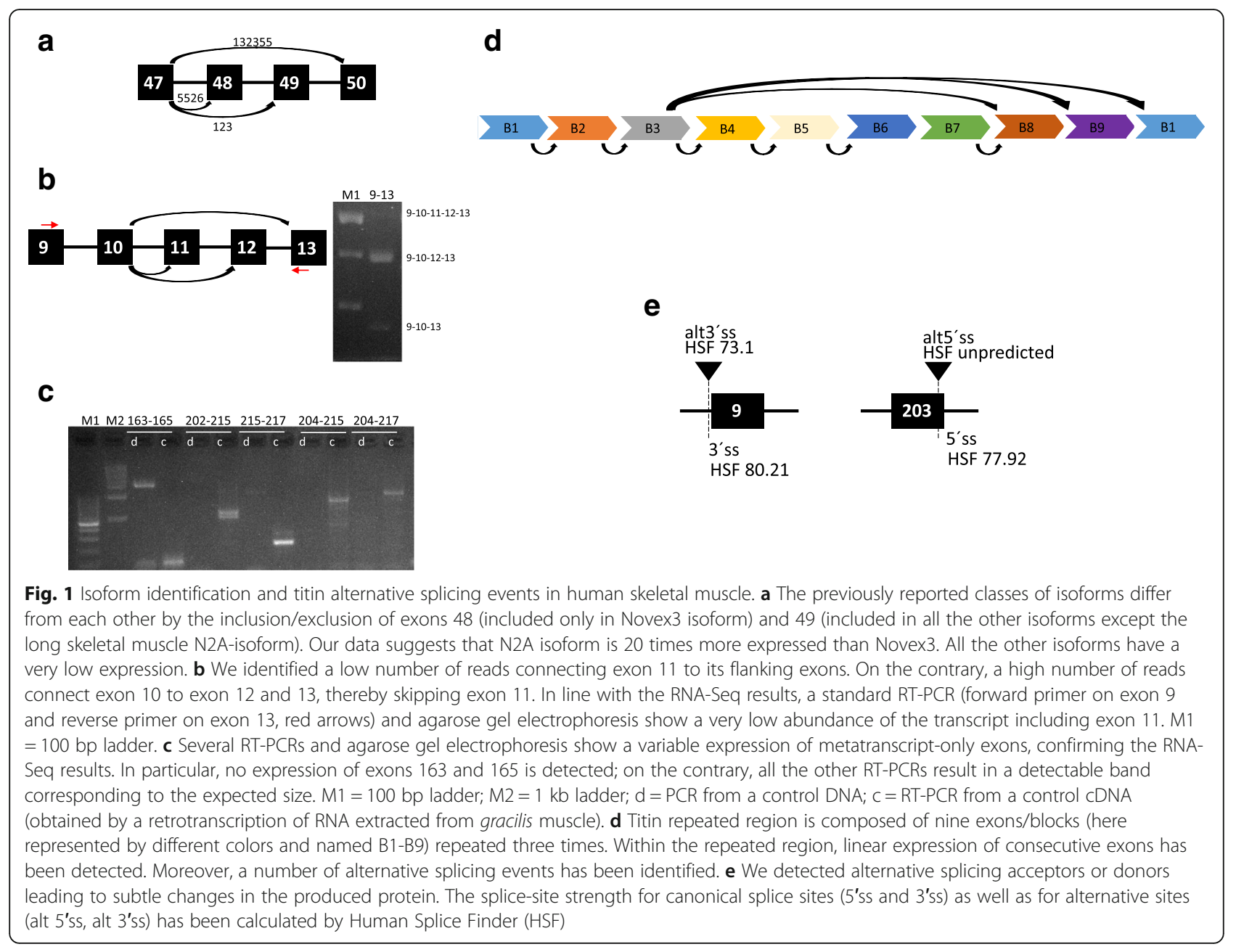

We then calculated the number of reads supporting each of the N2A canonical splicing events (Additional file 2: Table S2). Most of the canonical N2A junctions were identified. Interestingly, we noticed a very low number of reads supporting the inclusion of exon 11 (junctions linking exon 10-11 and exon 11-12). Similarly, we did not observe reads connecting exons 183 and 203.

Then, we proceeded to the analysis of the alternative splice events. We identified 4039 unique splicing events, most of them in one or a few samples and supported by a very low number of reads. In order to eliminate the background sequencing noise and/or very weakly expressed transcripts, we applied a stringent quality control (QC)-filtering process, prioritizing only splicing events $(n=498)$ supported by at least 1000 reads and identified in at least 14 samples. To reduce possible artefacts due to technical issues and obtain a less biased splicing pattern, we analyzed publicly available total mRNA sequencing data of adult gastrocnemius medialis from the ENCODE project. In a very conservative approach, we only focused on splicing events identified in our experimental samples as well as in the publicly available data (> 10 reads in ENCODE data).
After that, we proceeded with a multistep analysis, based on two different categories of splicing events: (1) ASEs involving canonical splice sites (out of the repeated region and within this area) and (2) ASEs involving alternative splicing sites.

1) We identified 46 unreported exon junctions, involving canonical splice sites out of the repeated region (Table 1). All these 46 ASEs are predicted to maintain the frame. For 23 ASEs, we performed RTPCR and all confirmed the RNA-Seq results.

We identified three ASEs (10-12; 10-13; 10-14), suggesting the skipping of exon 11 . In line with the RNA-Seq results, RT-PCR confirms that exon 11 was poorly expressed in human adult skeletal muscle (Fig. 1b).

Interestingly, 24 unreported junctions span metaonly exons, suggesting their partial inclusion in TTN human adult skeletal muscle transcripts (Table 1 and Fig. 1c).

We identified a high number of reads involving the canonical splice sites of exons included in the 
Table 1 Previously unreported junctions involving canonical splice sites out of the repeated region

\begin{tabular}{|c|c|c|c|}
\hline Donor exon & Acceptor exon & \#TotReads & \#TotReads encode \\
\hline 10 & 12 & 63,681 & 31,420 \\
\hline 10 & 13 & 37,864 & 22,425 \\
\hline 10 & 14 & 25,860 & 19,371 \\
\hline 36 & 38 & 2848 & 46 \\
\hline 51 & 54 & 2319 & 52 \\
\hline 85 & 88 & 8333 & 113 \\
\hline 112 & 114 & 10,267 & 45 \\
\hline 116 & 119 & 8651 & 1859 \\
\hline 132 & 134 & 16,926 & 2843 \\
\hline 137 & 143 & 1865 & 700 \\
\hline 144 & 146 & 8300 & 297 \\
\hline 146 & 151 & 10,262 & 805 \\
\hline 146 & 152 & 3145 & 778 \\
\hline 147 & 148 & 32,429 & 26,174 \\
\hline 148 & 149 & 26,771 & 12,941 \\
\hline 149 & 150 & 1304 & 951 \\
\hline 150 & 151 & 1857 & 2836 \\
\hline 153 & 158 & 12,300 & 116 \\
\hline 154 & 158 & 35,902 & 4086 \\
\hline 158 & 159 & 28,020 & 13,261 \\
\hline 158 & 167 & 1129 & 45 \\
\hline 158 & 168 & 3759 & 56 \\
\hline 158 & 171 & 2965 & 279 \\
\hline 158 & 172 & 7567 & 991 \\
\hline 159 & 167 & 3117 & 2355 \\
\hline 159 & 168 & 3192 & 1137 \\
\hline 159 & 171 & 3722 & 3283 \\
\hline 159 & 172 & 3627 & 1620 \\
\hline 167 & 168 & 1830 & 2517 \\
\hline 168 & 169 & 8111 & 7411 \\
\hline 169 & 170 & 5910 & 5129 \\
\hline 170 & 171 & 3237 & 8145 \\
\hline 171 & 172 & 1439 & 8414 \\
\hline 202 & 203 & 1358 & 15,787 \\
\hline 203 & 209 & 1463 & 14 \\
\hline 208 & 210 & 1741 & 27 \\
\hline 212 & 213 & 28,311 & 11,949 \\
\hline 213 & 214 & 8695 & 17,192 \\
\hline 214 & 215 & 13,360 & 12,752 \\
\hline 215 & 216 & 14,850 & 18,391 \\
\hline 215 & 217 & 1647 & 1208 \\
\hline 216 & 217 & 11,704 & 7775 \\
\hline
\end{tabular}

Table 1 Previously unreported junctions involving canonical splice sites out of the repeated region (Continued)

\begin{tabular}{llll}
\hline Donor exon & Acceptor exon & \#TotReads & \#TotReads encode \\
\hline 217 & 218 & 10,983 & 15,909 \\
219 & 222 & 1146 & 333 \\
224 & 226 & 3126 & 2401 \\
362 & 364 & 5458 & 2555 \\
\hline
\end{tabular}

Metatranscript-only exons in italics

repeated area (Additional file 3: Supplementary Material 1, Additional file 4: Table S3 and Additional file 5: Table S4). Well-known bias due to such repetitive regions hampers a comprehensive and accurate study of this region. However, our data suggests the linear expression of consecutive exons within this area. We also identified a number of ASEs linking non-consecutive exons within the repeated elements (Fig. 1d).

2) We observed the usage of alternative splice sites (acceptors or donors) located next to the canonical sites. Most of these alternative splice sites (16/19) would produce an in-frame insertion or deletion of a few amino acids. The Human Splicing Finder (HSF) program displayed high splice site scores for most of these alternative splice sites, further suggesting their real use in TTN transcripts (Table 2 and Fig. 1e).

Based on the aforementioned splicing events passing our stringent QC filters, we calculated for each of the coding exons showing an alternative splicing, and not included in the repeated region, the number of reads supporting their inclusion or exclusion in TTN transcripts and a subsequent inclusion value (Table 3). It is noteworthy that 13 meta-only exons are expressed but only 7 have an inclusion value higher than $10 \%$. On the other side, most of the canonical N2A exons, reported to be expressed in adult skeletal muscle, have a high inclusion value. Exon 11 as well as exons 155, 156, and 157 have an inclusion value lower than $50 \%$, indicating that they are mostly spliced out.

To evaluate the spatial and temporal expression of exon 11 and of meta-only exons, we examined a subset of publicly available RNA-Seq data from fetal skeletal muscles and fetal and adult hearts (Fig. 2). Interestingly, exon 11 is mostly expressed in fetal and adult hearts. Its expression is very low in adult and fetal skeletal muscles. Exon 148 has a similar expression in fetal and adult muscles, and it is mostly skipped in fetal and adult hearts. On the contrary, meta-only exons 213-217 are almost constitutively expressed in fetal muscles and their expression is halved in adult muscles. 
Table 2 List of events involving alternative splice sites

\begin{tabular}{|c|c|c|c|c|c|c|c|}
\hline Donor & Acceptor & \#Samples & \#Reads & Frame & $\begin{array}{l}\text { HSF consensus value } \\
\text { novel donor splice } \\
\text { site (value for wt) }\end{array}$ & $\begin{array}{l}\text { HSF consensus value } \\
\text { novel acceptor splice } \\
\text { site (value for wt) }\end{array}$ & \#Reads encode \\
\hline c.669 (ex5) & c.673 (ex6-alt acc) & 31 & 1195 & Yes & - & $78.86(85.41)$ & 287 \\
\hline c.1398 (ex8) & c.1399-3 (int8-alt acc) & 42 & 6992 & Yes & - & $73.1(80.21)$ & 4456 \\
\hline c.9471 (ex40) & c.9508 (ex41-alt acc) & 19 & 10,437 & Yes & - & $76.95(90.97)$ & 101 \\
\hline c.22528 (ex78) & c.22871 (ex80-alt acc) & 27 & 1287 & Yes & - & $\begin{array}{l}\text { Unpredicted } \\
(77.00)\end{array}$ & 198 \\
\hline c.29124 (ex102-alt don) & c.29228 (ex103-alt acc) & 31 & 3807 & No & Unpredicted (88.47) & $72.03(79.27)$ & 20 \\
\hline c.30754 (ex113) & c.30757 (ex114-alt acc) & 16 & 1537 & No & - & $72.87(85.71)$ & 15 \\
\hline c.31426 (ex118) & c.31433 (ex1 19-alt acc) & 31 & 4960 & Yes & - & $79.99(81.96)$ & 35 \\
\hline c.31762 (ex122) & c.31769 (ex123-alt acc) & 19 & 3455 & Yes & - & $86.28(67.88)$ & 33 \\
\hline c.32197 (ex127) & c.32207 (ex128-alt acc) & 25 & 1018 & Yes & - & $82.28(78.21)$ & 44 \\
\hline c.32392 (ex129) & c.32399 (ex130-alt acc) & 41 & 5055 & Yes & - & $82.29(75.58)$ & 121 \\
\hline c.33910 (ex145) & c.33917 (ex146-alt acc) & 18 & 1733 & Yes & - & $80.36(77.55)$ & 25 \\
\hline c.33994 (ex146) & c.34301 (ex148-alt acc) & 19 & 12,820 & Yes & - & $72.21(73.01)$ & 159 \\
\hline c.38058 (ex191-alt don) & c.39484 (ex208-alt acc) & 37 & 1038 & Yes & Unpredicted (76.37) & $74.68(80.08)$ & 32 \\
\hline c.38058 (ex191-alt don) & c.38980 (ex202-alt acc) & 37 & 1427 & Yes & Unpredicted (76.37) & $75.98(77.27)$ & 26 \\
\hline c.39063 (ex203-alt don) & c.39484 (ex208-alt acc) & 35 & 2300 & Yes & Unpredicted (77.92) & $74.68(80.08)$ & 29 \\
\hline c.39147 (ex204-alt don) & c.39484 (ex208-alt acc) & 41 & 4744 & Yes & Unpredicted (76.37) & $74.68(80.08)$ & 105 \\
\hline c.40786 (ex223) & c.40790 (ex224 - alt acc) & 30 & 1518 & Yes & - & $75.32(94.42)$ & 257 \\
\hline c.40876 (ex224) & c.40880 (ex225 - alt acc) & 24 & 1635 & Yes & - & $77.2(91.6)$ & 151 \\
\hline c.44646 (ex243-alt don) & c.44914 (ex245) & 20 & 10,706 & No & $83.39(82.15)$ & - & 15 \\
\hline
\end{tabular}

alt don alternative donor, alt acc alternative acceptor

A list of all detected ASEs that did not reach the minimum filtering criteria (i.e., a minimum of 14 out of 42 samples analyzed and at least 1000 supporting reads in total) or were not identified in the publicly available ENCODE data is included in Additional file 6: Table S5.

\section{Discussion}

Recent mRNA-Seq transcriptomic analyses show that most of multi-exonic genes are alternatively spliced [7, 10, 20]. In particular, a vast majority of ASEs are tissue specific [10], and skeletal muscle seems to be among the tissues showing the highest numbers of tissue-specific ASEs $[6,7,20]$.

Considering its 363 coding-exons and its genetic organization, a large number of ASEs were expected and partly reported in TTN transcripts. However, previous data, obtained by using different heterogeneous strategies in a pre-NGS era, did not provide a comprehensive view of the TTN splicing pattern and neither any unbiased repertoire of TTN ASEs in human adult skeletal muscles $[1,2,8]$.

In our study, by performing RNA-Seq analysis using 42 adult human skeletal muscle samples, we identified in a reliable way a large number of ASEs, some of them at a very high level.
We detected previously undescribed exon-exon junctions, suggesting novel, unreported skipping events. Exon 11 , included in the canonical adult skeletal muscle isoform $\mathrm{N} 2 \mathrm{~A}$, is mostly skipped in adult skeletal muscles. On the contrary, most of the so-called metatranscript-only exons are expressed in adult skeletal muscle at a variable level. Moreover, we identified alternative acceptors and donors leading to subtle changes in the produced protein. Although these events need to be experimentally validated, similar ASEs have already been described in other human genes and their functional relevance has been hypothesized [21-23].

With the exception of exon 11, the N-terminal exons, coding for the Z-disk part of titin, are mostly constitutively expressed. Exons 8 to 14 encode for seven copies of a specific domain, named Z-repeat $(\mathrm{Zr})$ [24]. In particular, exon 11 encodes for Z-repeat 4 [24], and its differential splicing has been previously reported [25]. Sorimachi and colleagues reported that Z-repeats 1, 2, 3, and 7 are expressed in all striated rabbit muscles, whereas the expression of $\mathrm{Zr} 4,5,6$ (corresponding to exons 11-12 and 13) is dependent on developmental stage and tissue-type [25]. The differential splicing of the titin Z-disk seems to be part of a larger and more complex process able to modulate Z-disk interactions via 
Table 3 Exon usage

\begin{tabular}{|c|c|c|c|c|c|c|c|c|c|}
\hline Exon(s) & $\begin{array}{l}\text { Inclusion } \\
\text { rate }\end{array}$ & $\begin{array}{l}\text { \#Inclusion } \\
\text { reads }\end{array}$ & $\begin{array}{l}\text { \#Exclusion } \\
\text { reads }\end{array}$ & $\begin{array}{l}\text { Skipping } \\
\text { event }\end{array}$ & Exon(s) & $\begin{array}{l}\text { Inclusion } \\
\text { rate }\end{array}$ & $\begin{array}{l}\text { \#lnclusion } \\
\text { reads }\end{array}$ & $\begin{array}{l}\text { \#Exclusion } \\
\text { reads }\end{array}$ & $\begin{array}{l}\text { Skipping } \\
\text { event }\end{array}$ \\
\hline ex1-10 & \multicolumn{4}{|c|}{ Constitutively expressed } & ex148 & $68 \%$ & 72,020 & 16,913 & $146-151 ; 146-152 ; 147-149$ \\
\hline ex11 & \multicolumn{4}{|c|}{ Constitutively spliced out } & ex149 & $67 \%$ & 53,414 & 13,407 & $146-151 ; 146-152$ \\
\hline ex12 & $54 \%$ & 147,944 & 63,724 & $10-13 ; 10-14$ & ex150 & $4 \%$ & 3161 & 35,240 & $146-151 ; 146-152 ; 149-151$ \\
\hline ex13 & $79 \%$ & 194,924 & 25,860 & $10-14$ & ex151 & $94 \%$ & 94,107 & 3145 & $146-152$ \\
\hline $\begin{array}{l}\text { ex14- } \\
36\end{array}$ & \multicolumn{4}{|c|}{ Constitutively expressed } & $\begin{array}{l}\text { ex152- } \\
153\end{array}$ & \multicolumn{4}{|c|}{ Constitutively expressed } \\
\hline ex37 & $98 \%$ & 335,403 & 2848 & $36-38$ & ex154 & $82 \%$ & 115,379 & 12,300 & $153-158$ \\
\hline \multirow{2}{*}{$\begin{array}{l}\text { ex38- } \\
44\end{array}$} & \multirow{2}{*}{\multicolumn{4}{|c|}{ Constitutively expressed }} & ex155 & $35 \%$ & 52,812 & \multirow[t]{3}{*}{48,202} & \multirow[t]{3}{*}{$153-158 ; 154-158$} \\
\hline & & & & & ex156 & $34 \%$ & 49,216 & & \\
\hline $\begin{array}{l}\text { ex45- } \\
46\end{array}$ & \multicolumn{4}{|c|}{ Constitutively spliced out } & ex157 & $40 \%$ & 64,075 & & \\
\hline ex47 & \multicolumn{4}{|c|}{ Constitutively expressed } & ex158 & \multicolumn{4}{|c|}{ Constitutively expressed } \\
\hline ex48 & $2 \%$ & 5526 & 132,355 & $47-50$ & ex 159 & $20 \%$ & 52,844 & 103,121 & \multirow{3}{*}{$\begin{array}{l}\text { 158-167;158-168;158- } \\
171 ; 158-172 ; 158-173 ; \\
158-175 ; 158-182 ; 158- \\
184 ; 158-191 ; 158-193 ; \\
158-204\end{array}$} \\
\hline ex49 & \multicolumn{4}{|c|}{ Constitutively spliced out } & & & & & \\
\hline $\begin{array}{l}\text { ex50- } \\
51\end{array}$ & \multicolumn{4}{|c|}{ Constitutively expressed } & & & & & \\
\hline ex52 & $98 \%$ & 222,051 & 2319 & $51-54$ & $\begin{array}{l}\text { ex160- } \\
\text { ex166 }\end{array}$ & \multicolumn{4}{|c|}{ Constitutively spliced out } \\
\hline ex53 & $98 \%$ & 252,059 & & & ex 167 & $2 \%$ & 6076 & 123,699 & \multirow{4}{*}{$\begin{array}{l}158-168 ; 158-171 ; 158- \\
172 ; 158-173 ; 158-175 ; 158- \\
182 ; 158-184 ; 158-191 ; 158- \\
193 ; 158-204 ; 159-168 ; 159- \\
171 ; 159-172 ; 159-173 ; 159- \\
175 ; 159-184 ; 159-193\end{array}$} \\
\hline $\begin{array}{l}\text { ex54- } \\
\text { ex78 }\end{array}$ & \multicolumn{4}{|c|}{ Constitutively expressed } & & & & & \\
\hline ex79 & $99 \%$ & 262,245 & 1287 & c. $22,528-22,871$ & & & & & \\
\hline ex80- & \multirow{2}{*}{\multicolumn{4}{|c|}{ Constitutively expressed }} & & & & & \\
\hline & & & & & ex 168 & $7 \%$ & 16,892 & 116,748 & \multirow{4}{*}{$\begin{array}{l}158-171 ; 158-172 ; 158- \\
173 ; 158-175 ; 158-182 ; 158- \\
184 ; 158-191 ; 158-193 ; 158- \\
204 ; 159-168 ; 159-171 ; 159- \\
172 ; 159-173 ; 159-175 ; 159- \\
184 ; 159-193\end{array}$} \\
\hline ex86 & $91 \%$ & 174,217 & \multirow[t]{3}{*}{8333} & \multirow[t]{3}{*}{$85-88$} & ex 169 & $6 \%$ & 14,021 & & \\
\hline ex87 & $93 \%$ & 216,577 & & & ex 170 & $4 \%$ & 9147 & & \\
\hline $\begin{array}{l}\text { ex88- } \\
112\end{array}$ & \multicolumn{2}{|c|}{ Constitutively expressed } & & & & & & & \\
\hline ex113 & $90 \%$ & 187,870 & 10,267 & $112-114$ & ex 171 & $5 \%$ & 11,363 & 110,061 & \multirow{4}{*}{$\begin{array}{l}158-172 ; 158-173 ; 158- \\
175 ; 158-182 ; 158-184 ; 158- \\
191 ; 158-193 ; 158-204 ; 159- \\
172 ; 159-173 ; 159-175 ; 159- \\
184 ; 159-193\end{array}$} \\
\hline $\begin{array}{l}\text { ex114- } \\
116\end{array}$ & \multicolumn{4}{|c|}{ Constitutively expressed } & & & & & \\
\hline ex117 & $92 \%$ & 200,426 & 8651 & $116-119$ & & & & & \\
\hline ex118 & $92 \%$ & 209,844 & & & $\begin{array}{l}\text { ex172- } \\
205\end{array}$ & Repeated & region & & \\
\hline $\begin{array}{l}\text { ex119- } \\
132\end{array}$ & \multicolumn{4}{|c|}{ Constitutively expressed } & ex206 & $81 \%$ & 184,735 & 21,799 & 175-209;184-209;с.38058- \\
\hline ex133 & $66 \%$ & 66,307 & 16,926 & $132-134$ & ex207 & $68 \%$ & 91,407 & & $\begin{array}{l}\text { C.39484; } 193-20 ; C .39003- \\
\text { c.39484;203-209;с.39147- }\end{array}$ \\
\hline ex134- & Constituti & vely expresse & & & & & & & C.39484 \\
\hline & & & 1865 & & ex208 & $76 \%$ & 86,726 & 13,717 & $\begin{array}{l}175-209 ; 184-209 ; 193- \\
209 ; 203-209\end{array}$ \\
\hline ex139 & $96 \%$ & 86,065 & & & ex209 & $97 \%$ & 99,982 & 1741 & $208-210$ \\
\hline ex140 & $95 \%$ & 77,132 & & & $\begin{array}{l}\text { ex210- } \\
212\end{array}$ & Constituti & ely expresse & & \\
\hline ex141 & $96 \%$ & 97,228 & & & ex213 & $26 \%$ & 37,006 & 53,547 & $212-218$ \\
\hline ex142 & $96 \%$ & 100,706 & & & $e \times 214$ & $17 \%$ & 22,055 & & \\
\hline $\begin{array}{l}\text { ex143- } \\
144\end{array}$ & Constituti & vely expresse & & & $e \times 215$ & $22 \%$ & 29,857 & & \\
\hline ex145 & $83 \%$ & 82,433 & 8300 & $144-146$ & $e \times 216$ & $19 \%$ & 26,554 & 55,194 & $212-218 ; 215-217$ \\
\hline ex146 & Constituti & vely expresse & & & ex217 & $19 \%$ & 24,334 & 53,547 & $212-218$ \\
\hline ex147 & $62 \%$ & 86,205 & 26,227 & $\begin{array}{l}\text { c.33994-34,301;146- } \\
151 ; 146-152\end{array}$ & $\begin{array}{l}\text { ex218- } \\
\text { ex219 }\end{array}$ & Constituti & ely expresse & & \\
\hline
\end{tabular}

Table 3 Exon usage (Continued)

ex160- Constitutively spliced out 172;158-173;158-175;158(58-184;158-191;158$58-171 ; 158-172 ; 158$ 72;159-173;159-175;15958-172:158-173:15872;159-173;159-175;159- 
Table 3 Exon usage (Continued)

\begin{tabular}{|c|c|c|c|c|}
\hline Exon(s) & $\begin{array}{l}\text { Inclusion } \\
\text { rate }\end{array}$ & $\begin{array}{l}\text { \#Inclusion } \\
\text { reads }\end{array}$ & $\begin{array}{l}\text { \#Exclusion } \\
\text { reads }\end{array}$ & $\begin{array}{l}\text { Skipping } \\
\text { event }\end{array}$ \\
\hline ex220 & $99 \%$ & 168,189 & 1146 & $219-222$ \\
\hline ex221 & $99 \%$ & 194,654 & & \\
\hline $\begin{array}{l}\text { ex222- } \\
224\end{array}$ & \multicolumn{4}{|c|}{ Constitutively expressed } \\
\hline ex225 & $95 \%$ & 124,798 & 3126 & $224-226$ \\
\hline $\begin{array}{l}\text { ex226- } \\
243\end{array}$ & \multicolumn{4}{|c|}{ Constitutively expressed } \\
\hline ex244 & $93 \%$ & 285,487 & 10,706 & c. $44,646-44,914$ \\
\hline $\begin{array}{l}\text { ex245- } \\
362\end{array}$ & \multicolumn{4}{|c|}{ Constitutively expressed } \\
\hline ex363 & $91 \%$ & 115,672 & 5458 & $362-364$ \\
\hline ex364 & \multicolumn{4}{|c|}{ Constitutively expressed } \\
\hline
\end{tabular}

Metatranscript-only exons in italics

splicing regulation. The $\mathrm{N}$-terminal Z-disk region of titin binds a number of proteins, including alpha-actinin, nebulin, and filamin $\mathrm{C}$ that undergo a similar process of differential splicing [26-28].

As expected, most of the ASEs occur in the I-band region of titin, where a large number of exons are alternatively spliced [3, 4]. It is noteworthy that exon 148, thought to be a meta-only exon, has an inclusion rate comparable to that of its neighboring exons in both adult and fetal skeletal muscles. Moreover, our experimental data as well as publicly available data suggests a significant expression of the meta-only exons $213,214,215,216$, and 217 in adult skeletal muscle, although their inclusion is higher in fetal muscles. In the M-band, we identified the previously reported splicing event (skipping of exon 363), producing the so called is7- and is7+ isoforms [29, 30]. In line with previous data, exon 363 is skipped in about $10 \%$ of TTN transcripts in human adult skeletal muscle.

As already discussed for the Z-disk splicing events, the regulation of alternative splicing events probably corresponds to modulation of interaction networks. For example, it is well known that the alternatively spliced is7 region, encoded by exon 363 , binds the calcium-dependent protease calpain 3 (CAPN3) [31]. On the other hand, the role of the titin, and also nebulin, filament length (as a result of splicing events) on the sarcomere length and its passive elastic properties is still under debate [32-34].

Mutations in the TTN gene cause several different and heterogeneous skeletal muscle disorders with or without cardiac involvement, characterized by a variability in the age of onset, muscle involvement, and disease-course $[11,12,35]$. In addition, truncating mutations (TTNtv) have been associated with dilated cardiomyopathy (DCM) $[13,14]$. A genotype-phenotype correlation has been observed to some extent [11, 15]. Mutations in metatranscript-only exons have recently been associated with a congenital titinopathy, characterized by arthrogryposis multiplex congenita and severe axial hypotonia as a form of congenital amyoplasia without cardiac involvement [36]. The hypothesis is that metatranscript-only mutations (mostly truncating mutations) specifically and selectively affect developmental isoforms, leading to a
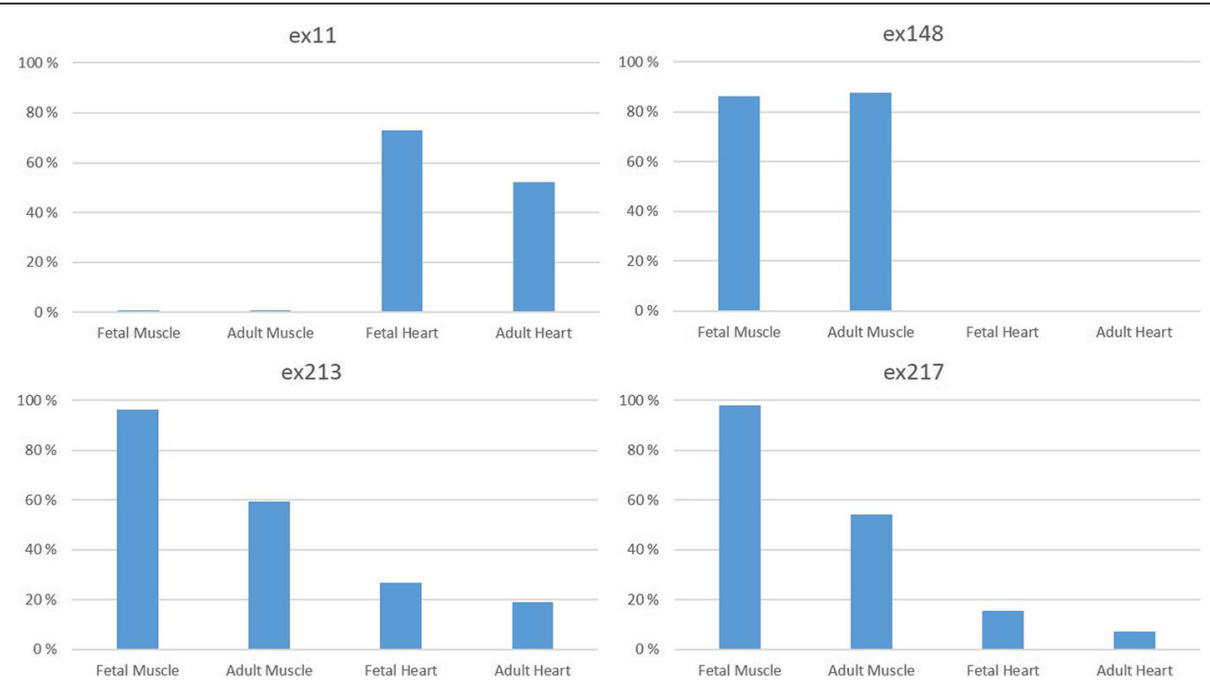

Fig. 2 Comparison of alternative splicing events among different tissues at different developmental stages. The analysis of publicly available total mRNA sequencing data from the ENCODE project shows that exon 11 is expressed only in cardiac muscles, whereas the expression of exon 148 is limited to skeletal muscles. Exons 213 and 217 show an increased expression in fetal skeletal (and, at least in part, cardiac) muscle compared to the adult expression. The reported values correspond to the inclusion values, based on the number of reads supporting each exon inclusion or exclusion in TTN transcripts 
prenatal or congenital phenotype with a stable postnatal disease-course or weakness amelioration. On the contrary, proximal truncating mutations in canonical exons expressed on both alleles in adult isoforms lead to a premature truncated protein with nonsense mediated decay and would probably cause fetal death. The pathogenesis of TTNtv-related cardiomyopathies is probably more unclear; their penetrance is markedly reduced and they show a positional effect [14]. In particular, only TTNtv occurring in constitutive exons are significantly associated with DCM [14].

Deciphering the effective expression pattern of each TTN-exon, including meta-only exons, is crucial for a better understanding of TTN-related disorders. Our data clearly shows a variable expression for most of the metaonly exons $(148,150,159,167-171,213-217)$, confirming, however, that some of them (160-166) are not expressed at all in human adult skeletal muscles. Our findings suggest the need for a more careful interpretation of the variants identified in a clinical setting.

Here, we provided an accurate inventory of ASEs in human adult skeletal muscles, which suggest the presence of a high number of undescribed isoforms. Moreover, taking into account all the alternative splicing events occurring in $T T N$, we calculated a reliable inclusion value for titin exons.

Further work remains to be done in order to refine our results. Long-read sequencing technologies, for example, will allow the identification of multiple splicing events along the same molecule, thereby elucidating how the individual splice events here described are connected, and thus confirming the presence of unreported isoforms. Similarly, a larger number of samples from each skeletal muscle type has to be analyzed in order to identify muscle-type specific ASEs or splicing patterns, considering that the current experimental setting has not identified any clear splicing difference among the muscles analyzed (Additional file 7: Table S6).

The exonic usage and the subsequent isoform expression seem to be finely regulated among different developmental and physiological and/or pathological states $[2,17,37]$. A further refinement of TTN expression profiling in different tissues and/or different physiological and pathological states (including regenerating or injured muscles) would be of a great clinical relevance, deepening, for example, our understanding of the role of TTN variants in complex human diseases.

\section{Conclusions}

We have identified and partly characterized a large number of alternative splicing events in titin, providing the first RNA-Seq-based, accurate and comprehensive picture of TTN splicing pattern in adult human skeletal muscle. This same approach will probably unveil similar complex splicing patterns for other muscle transcripts.

\section{Additional files}

Additional file 1: Table S1. List of samples analyzed. (XLSX $11 \mathrm{~kb}$ )

Additional file 2: Table S2. N2A splicing junctions. (XLSX 44 kb)

Additional file 3: Supplementary Material 1: Titin repeated region. (DOCX $112 \mathrm{~kb}$ )

Additional file 4: Table S3. Previously reported junctions in the repeated region. (XLSX $11 \mathrm{~kb}$ )

Additional file 5: Table S4. Unreported junctions involving exons in the repeated region. (XLSX $12 \mathrm{~kb})$

Additional file 6: Table S5. List of alternative splicing events not reaching the minimum filtering criteria or not identified in the publicly available ENCODE data. (XLSX $155 \mathrm{~kb}$ )

Additional file 7: Table S6. Previously unreported junctions clustered accordingly to specific skeletal muscle types. (XLSX 17 kb)

\section{Abbreviations \\ AS: Alternative splicing; ASEs: Alternative splicing events; HSF: Human Splicing Finder; NGS: Next-generation sequencing; PCR: Polymerase chain reaction; QC: Quality control; RNA-Seq: RNA sequencing; RT-PCR: Reverse transcriptase-polymerase chain reaction; TTNtv: Titin truncating variants}

\section{Acknowledgements}

The authors would like to thank Meharji Arumilli for his advice in the analysis of RNA-Seq data.

\section{Funding}

This study was supported by Finnish Academy, Juselius Research Foundation, Association Française contre les Myopathies and Orion Research Foundation. The funders had no role in the study design, data collection and analysis, decision to publish, or preparation of the manuscript.

\section{Availability of data and materials}

All data generated or analyzed during this study are included in this published article and its supplementary information files. Raw data is available from the corresponding author on reasonable request.

\section{Authors' contributions}

All authors participated in designing all the studies. SH collected skeletal muscles. PHJ, SH, LP, PA, and PH conducted the RNA-sequencing. MS and $\mathrm{PHJ}$ analyzed the RNA-sequencing results and performed the experimental validation. $\mathrm{MS}, \mathrm{PHJ}, \mathrm{BU}$, and $\mathrm{PH}$ wrote the manuscript. All authors have been involved with reviewing the manuscript and have approved the final version.

Ethics approval and consent to participate

A written informed consent was signed by all the patients and the Tampere University Hospital (Tampere, Finland) Ethics Committee approved the study.

Consent for publication

Not applicable

Competing interests

The authors declare that they have no competing interests.

\section{Publisher's Note}

Springer Nature remains neutral with regard to jurisdictional claims in published maps and institutional affiliations.

\section{Author details}

${ }^{1}$ Folkhälsan Research Center, University of Helsinki, Helsinki, Finland. ${ }^{2}$ Department of Pathology, Fimlab Laboratories, Tampere University Hospital, Tampere, Finland. Institute of Biotechnology, University of Helsinki, Helsinki, Finland. ${ }^{4}$ Vaasa Central Hospital, Vaasa, Finland. ${ }^{5}$ Folkhälsan Institute of Genetics, Department of Medical Genetics, University of Helsinki, Biomedicum, Haartmaninkatu 8, Pb 63, 00014 Helsinki, Finland. 
Received: 7 December 2017 Accepted: 5 March 2018 Published online: 29 March 2018

\section{References}

1. Bang M-L, Centner T, Fornoff F, Geach AJ, Gotthardt M, McNabb M, Witt CC, Labeit D, Gregorio CC, Granzier H, et al. The complete gene sequence of titin, expression of an unusual approximately 700-kDa titin isoform, and its interaction with obscurin identify a novel Z-line to I-band linking system. Circ Res. 2001;89(11):1065-72.

2. Guo W, Bharmal SJ, Esbona K, Greaser ML. Titin diversity -alternative splicing gone wild. J Biomed Biotechnol. 2010;2010:753675.

3. Linke WA, Rudy DE, Centner T, Gautel M, Witt C, Labeit S, Gregorio CC. I-band titin in cardiac muscle is a three-element molecular spring and is critical for maintaining thin filament structure. J Cell Biol. 1999;146(3):631-44.

4. Freiburg A, Trombitas K, Hell W, Cazorla O, Fougerousse F, Centner T, Kolmerer B, Witt C, Beckmann JS, Gregorio CC, et al. Series of exon-skipping events in the elastic spring region of titin as the structural basis for myofibrillar elastic diversity. Circ Res. 2000;86(11):1114-21.

5. Labeit S, Lahmers S, Burkart C, Fong C, McNabb M, Witt S, Witt C, Labeit D, Granzier $\mathrm{H}$. Expression of distinct classes of titin isoforms in striated and smooth muscles by alternative splicing, and their conserved interaction with filamins. J Mol Biol. 2006:362(4):664-81.

6. Castle JC, Zhang C, Shah JK, Kulkarni AV, Kalsotra A, Cooper TA, Johnson JM. Expression of 24,426 human alternative splicing events and predicted cis regulation in 48 tissues and cell lines. Nat Genet. 2008;40(12):1416-25.

7. Brinegar AE, Xia Z, Loehr JA, Li W, Rodney GG, Cooper TA. Extensive alternative splicing transitions during postnatal skeletal muscle development are required for calcium handling functions. Elife. 2017;6. https://doi.org/10.7554/eLife.27192.

8. Greaser ML, Krzesinski PR, Warren CM, Kirkpatrick B, Campbell KS, Moss RL. Developmental changes in rat cardiac titin/connectin: transitions in normal animals and in mutants with a delayed pattern of isoform transition. J Muscle Res Cell Motil. 2005:26(6-8):325-32.

9. Wang Z, Gerstein M, Snyder M. RNA-Seq: a revolutionary tool for transcriptomics. Nat Rev Genet. 2009;10(1):57-63.

10. Wang ET, Sandberg R, Luo S, Khrebtukova I, Zhang L, Mayr C, Kingsmore SF, Schroth GP, Burge CB. Alternative isoform regulation in human tissue transcriptomes. Nature. 2008;456(7221):470-6.

11. Savarese M, Sarparanta J, Vihola A, Udd B, Hackman P. Increasing role of titin mutations in neuromuscular disorders. J Neuromuscul Dis. 2016;3(3):293-308.

12. Chauveau C, Rowell J, Ferreiro A. A rising titan: $\pi T N$ review and mutation update. Hum Mutat. 2014;35(9):1046-59.

13. Herman DS, Lam L, Taylor MR, Wang L, Teekakirikul P, Christodoulou D, Conner L, DePalma SR, McDonough B, Sparks E, et al. Truncations of titin causing dilated cardiomyopathy. N Engl J Med. 2012;366(7):619-28.

14. Schafer $\mathrm{S}$, de Marvao A, Adami E, Fiedler LR, Ng B, Khin E, Rackham OJ, van Heesch S, Pua CJ, Kui M, et al. Titin-truncating variants affect heart function in disease cohorts and the general population. Nat Genet. 2017:49(1):46-53.

15. Hackman P, Udd B, Bonnemann CG, Ferreiro A, Titinopathy Database C. 219th ENMC International Workshop Titinopathies International database of titin mutations and phenotypes, Heemskerk, The Netherlands, 29 April-1 May 2016. Neuromuscul Disord. 2017;27(4):396-407.

16. Evila A, Palmio J, Vihola A, Savarese M, Tasca G, Penttila S, Lehtinen S, Jonson PH, De Bleecker J, Rainer P, et al. Targeted next-generation sequencing reveals novel TTN mutations causing recessive distal titinopathy. Mol Neurobiol. 2017;54(9): 7212-23. https://doi.org/10.1007/s12035-016-0242-3.

17. Huovinen S, Penttila S, Somervuo P, Keto J, Auvinen P, Vihola A, Huovinen S, Pelin K, Raheem O, Salenius J, et al. Differential isoform expression and selective muscle involvement in muscular dystrophies. Am J Pathol. 2015; 185(10):2833-42.

18. Kim D, Pertea G, Trapnell C, Pimentel H, Kelley R, Salzberg SL. TopHat2: accurate alignment of transcriptomes in the presence of insertions, deletions and gene fusions. Genome Biol. 2013:14(4):R36.

19. Desmet FO, Hamroun D, Lalande M, Collod-Beroud G, Claustres M, Beroud C. Human splicing finder: an online bioinformatics tool to predict splicing signals. Nucleic Acids Res. 2009;37(9):e67.

20. Llorian M, Smith CW. Decoding muscle alternative splicing. Curr Opin Genet Dev. 2011;21(4):380-7.

21. Koren E, Lev-Maor G, Ast G. The emergence of alternative $3^{\prime}$ and $5^{\prime}$ splice site exons from constitutive exons. PLoS Comput Biol. 2007;3(5):e95.
22. Bouge AL, Murauer E, Beyne E, Miro J, Varilh J, Taulan M, Koenig M, Claustres M, Tuffery-Giraud S. Targeted RNA-Seq profiling of splicing pattern in the DMD gene: exons are mostly constitutively spliced in human skeletal muscle. Sci Rep. 2017:7:39094.

23. Yan X, Sablok G, Feng G, Ma J, Zhao H, Sun X. nagnag: identification and quantification of NAGNAG alternative splicing using RNA-Seq data. FEBS Lett. 2015;589(15):1766-70.

24. Gautel M, Goulding D, Bullard B, Weber K, Furst DO. The central Z-disk region of titin is assembled from a novel repeat in variable copy numbers. Cell Sci. 1996;109(Pt 11):2747-54.

25. Sorimachi H, Freiburg A, Kolmerer B, Ishiura S, Stier G, Gregorio CC, Labeit D, Linke WA, Suzuki K, Labeit S. Tissue-specific expression and alpha-actinin binding properties of the Z-disc titin: implications for the nature of vertebrate Z-discs. J Mol Biol. 1997;270(5):688-95.

26. Laitila J, Hanif M, Paetau A, Hujanen S, Keto J, Somervuo P, Huovinen S, Udd B, Wallgren-Pettersson C, Auvinen P, et al. Expression of multiple nebulin isoforms in human skeletal muscle and brain. Muscle Nerve. 2012:46(5):730-7.

27. van der Flier A, Kuikman I, Kramer D, Geerts D, Kreft M, Takafuta T, Shapiro SS, Sonnenberg A. Different splice variants of filamin-B affect myogenesis, subcellular distribution, and determine binding to integrin [beta] subunits. J Cell Biol. 2002;156(2):361-76.

28. Parr T, Waites GT, Patel B, Millake DB, Critchley DR. A chick skeletal-muscle alpha-actinin gene gives rise to two alternatively spliced isoforms which differ in the EF-hand $\mathrm{Ca}(2+)-$ binding domain. Eur J Biochem. 1992;210(3):801-9.

29. Kolmerer B, Olivieri N, Witt CC, Herrmann BG, Labeit S. Genomic organization of $\mathrm{M}$ line titin and its tissue-specific expression in two distinct isoforms. J Mol Biol. 1996;256(3):556-63.

30. Charton K, Suel L, Henriques SF, Moussu JP, Bovolenta M, Taillepierre M, Becker C, Lipson K, Richard I. Exploiting the CRISPR/Cas9 system to study alternative splicing in vivo: application to titin. Hum Mol Genet. 2016;25(20):4518-32.

31. Sorimachi H, Kinbara K, Kimura S, Takahashi M, Ishiura S, Sasagawa N, Sorimachi N, Shimada H, Tagawa K, Maruyama K. Muscle-specific calpain, p94, responsible for limb girdle muscular dystrophy type 2A, associates with connectin through IS2, a p94-specific sequence. J Biol Chem. 1995;270(52):31158-62.

32. Kolb J, Li F, Methawasin M, Adler M, Escobar YN, Nedrud J, Pappas CT, Harris $\mathrm{SP}$, Granzier $\mathrm{H}$. Thin filament length in the cardiac sarcomere varies with sarcomere length but is independent of titin and nebulin. J Mol Cell Cardiol. 2016;97:286-94.

33. Labeit S, Ottenheijm CA, Granzier H. Nebulin, a major player in muscle health and disease. FASEB J. 2011;25(3):822-9.

34. Castillo A, Nowak R, Littlefield KP, Fowler VM, Littlefield RS. A nebulin ruler does not dictate thin filament lengths. Biophys J. 2009;96(5):1856-65.

35. Gerull B. The rapidly evolving role of titin in cardiac physiology and cardiomyopathy. Can J Cardiol. 2015:31(11):1351-9.

36. Fernandez-Marmiesse A, Carrascosa-Romero MC, Alfaro Ponce B, Nascimento A, Ortez C, Romero N, Palacios L, Jimenez-Mallebrera C, Jou C, Gouveia S, et al. Homozygous truncating mutation in prenatally expressed skeletal isoform of TTN gene results in arthrogryposis multiplex congenita and myopathy without cardiac involvement. Neuromuscul Disord. 2017;27(2):188-92.

37. Braun T, Gautel M. Transcriptional mechanisms regulating skeletal muscle differentiation, growth and homeostasis. Nat Rev Mol Cell Biol. 2011;12(6):349-61.

\section{Submit your next manuscript to BioMed Central and we will help you at every step:}

- We accept pre-submission inquiries

- Our selector tool helps you to find the most relevant journal

- We provide round the clock customer support

- Convenient online submission

- Thorough peer review

- Inclusion in PubMed and all major indexing services

- Maximum visibility for your research

Submit your manuscript at www.biomedcentral.com/submit
C) Biomed Central 